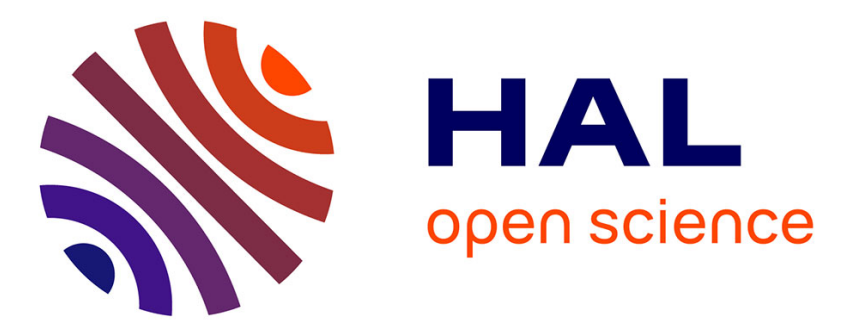

\title{
Solid State NMR Studies of Intact Lipopolysaccharide Endotoxin
}

Cédric Laguri, Alba Silipo, Alessandra M Martorana, Paul Schanda, Roberta Marchetti, Alessandra Polissi, Antonio Molinaro, Jean-Pierre Simorre

\section{- To cite this version:}

Cédric Laguri, Alba Silipo, Alessandra M Martorana, Paul Schanda, Roberta Marchetti, et al.. Solid State NMR Studies of Intact Lipopolysaccharide Endotoxin. ACS Chemical Biology, 2018, 13 (8), pp.2106 - 2113. 10.1021/acschembio.8b00271 . hal-01875923

\section{HAL Id: hal-01875923 \\ https://hal.univ-grenoble-alpes.fr/hal-01875923}

Submitted on 22 Oct 2021

HAL is a multi-disciplinary open access archive for the deposit and dissemination of scientific research documents, whether they are published or not. The documents may come from teaching and research institutions in France or abroad, or from public or private research centers.
L'archive ouverte pluridisciplinaire HAL, est destinée au dépôt et à la diffusion de documents scientifiques de niveau recherche, publiés ou non, émanant des établissements d'enseignement et de recherche français ou étrangers, des laboratoires publics ou privés. 
Solid State NMR studies of intact lipopolysaccharide endotoxin

Cedric Laguri ${ }^{1}$, Alba Silipo ${ }^{2}$, Alessandra M. Martorana ${ }^{3}$, Paul Schanda ${ }^{1}$, Roberta Marchetti ${ }^{2}$, Alessandra Polissi ${ }^{3}$, Antonio Molinaro ${ }^{2, *}$ and Jean-Pierre Simorre ${ }^{1, *}$

${ }^{1}$ Univ. Grenoble Alpes, CNRS, CEA, IBS, F-38000 Grenoble

${ }^{2}$ University of Naples Federico II, Department of Chemical Sciences, via cintia 4, Napoli, Italy

${ }^{3}$ University of Milano, Department of Pharmacological and Biomolecular Sciences, Via Balzaretti 9, Milano, Italy

*Correspondence: jean-pierre.simorre@ibs.fr (JP. S.) and molinaro@unina.it (A. M.)

\section{ABSTRACT}

Lipopolysaccharides (LPS) are complex glycolipids forming the outside layer of gram-negative bacteria. Their hydrophobic and heterogeneous nature greatly hampers their structural study in an environment similar to the bacterial surface. We have studied LPS purified from $E$. coli and pathogenic $P$. aeruginosa with long O-antigen polysaccharides assembled in solution as vesicles or elongated micelles. Solid-state NMR with Magic-angle spinning permitted the identification of NMR signals arising from regions with different flexibilities in the LPS, from the lipid components to the O-antigen polysaccharides. Atomic scale data on the LPS enabled to study the interaction of gentamicin antibiotic bound to $P$. aeruginosa LPS for which we could confirm that a specific oligosaccharide is involved in the antibiotic binding. The possibility to study LPS alone and bound to a ligand when it is assembled in membrane like structures opens great prospects for the investigation of proteins and antibiotics that specifically target such an important molecule at the surface of gram-negative bacteria.

\section{INTRODUCTION}

Gram negative bacteria lipopolysaccharides (LPSs) are key microbial macromolecules involved in a plethora of functions in prokaryotes and in the elicitation of innate immune response in insects, 
animals and plants ${ }^{1,2}$. Gram negative microbes possess a double system of membranes (Figure 1) in which LPSs are only present on the outer leaflet of the external membrane but in great abundance.

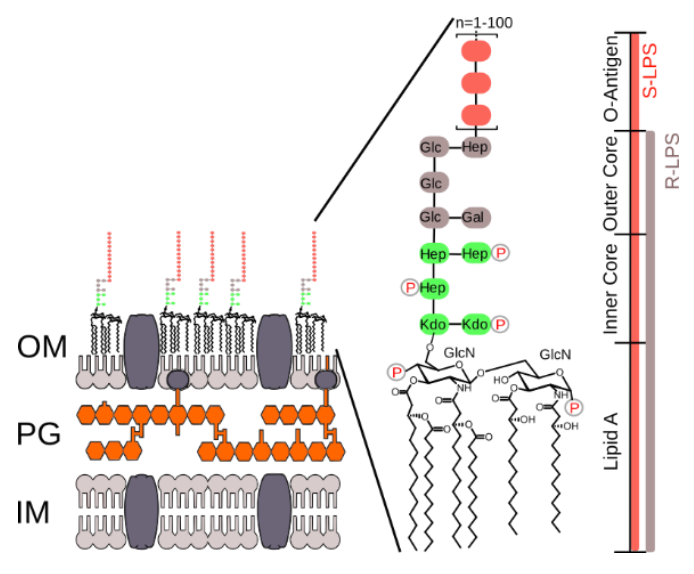

Figure 1 Localization and structure of lipopolysaccharides. LPSs constitute the major component of outer-membrane (OM) external leaflet of gram-negative bacteria (left). LPS is composed of three moieties, the lipid A, core oligosaccharides and O-antigens(right). PG Peptidoglycan, IM Innermembrane.

LPS is composed of a highly conserved hydrophobic lipid A moiety, a core oligosaccharide (core OS), and in many species a long-chain O-antigenic polysaccharide (Figure 1). Gram negative bacteria are more resistant to antibiotic treatment mainly due to their highly impermeable outer membrane where LPS is considered as the key actor in the membrane integrity ${ }^{3}$. Indeed, the LPS layer is highly ordered and is stabilized by electrostatic interactions between divalent cations (as $\mathrm{Ca}^{2+}$ and $\mathrm{Mg}^{2+}$ ) and phosphate groups of the lipid A part. This low fluidity of the layer confers to the outer membrane a low permeability to hydrophobic compounds and to higher molecular weight hydrophilic compounds. Only certain antibiotics directed against Gram-negative bacteria, such as polymyxin B or gentamicin, are able to destabilize the outer membrane leading to the disruption of the membrane integrity ${ }^{4}$. LPS is also a bacterial hallmark, a microbe associated molecular pattern, which is specifically recognized by eukaryotic immunity cell line receptors as a microbial signature ${ }^{1,2}$. For these organisms, this preliminary step of the innate immunity process, serves to trigger the response in case of pathogenicity or to shut down the response itself in case of beneficial interactions, such as 
symbiosis. In animals/humans, LPS is extracellularly detected by its lipid A ${ }^{5}$ at the level of immunity cell lines by a binary protein system (TLR4/MD2) which upon physical binding triggers the downstream reaction cascade ${ }^{1}$. Moreover, caspase-4/5 in humans and caspase-11 in mice detect intracellular LPS thus, likely being in direct binding and detection of intracellular pathogens ${ }^{6}$.

To understand these different biological processes at the molecular level, it is important to have methods to characterize macromolecular organization formed by LPSs in the membrane or in interaction with other proteins and/or ligands. However, because of their glycolipid nature they are generally neither soluble in water nor soluble in any organic solvents hampering the structural and functional characterization of LPSs in their intact state. The determination of the primary structure of a LPS is nowadays carried out separately on lipid A and O-antigen, chemically split in advance, by means of chemical analysis and biophysical methodologies (Gas Liquid Chromatography coupled to Mass spectrometry, GLC-MS; Mass Spectrometry, MS and solution-state Nuclear Magnetic Resonance, NMR $)^{7,8}$. Intact LPS molecules have been previously investigated, some of them also by NMR but no systematic approach has been adopted so far $^{9-11}$. The most exhaustive NMR analysis of intact LPS molecules including 3D structure determination and dynamics were conducted with LPS solubilized in detergent micelles ${ }^{12,13}$.

While these methods are efficient in attaining chemical structural studies of LPSs, there is a lack of alternative approaches able to study interaction of key molecules (receptors/antibiotics) with membrane surfaces formed by LPS. In fact, it is very important in this perspective to have a thorough view at molecular level at the whole intact molecule, at its overall supramolecular structure, at its delivery and at its atomic epitopes when bound and/or recognized by other ligands.

Atomic scale analysis of cell-surface glycoconjugates is particularly challenging. Magic Angle Spinning (MAS) solid state NMR (ssNMR) has provided in the last years interesting information on complex macromolecules alone or in complex with ligands (ions/proteins) ${ }^{14-18}$.

Therefore, we have deemed that ssNMR could provide the missing information on the different supramolecular organizations adopted by these molecules and we have undertaken the study of intact 
LPS in an environment close to the bacterial membrane. To this aim, in the following work we studied LPS extracted from different bacterial strains and with the two different molecular arrangements, a rough type LPS (a lipooligosaccharide, R-LPS) from E. coli K-12 and a smooth-type LPS (S-LPS) from $P$. aeruginosa PAO1 strains (Figure 1). The analysis of LPSs by ssNMR indeed enabled to observe the main characteristics of both LPS molecules, i.e., comparable to the data obtained when LPS is chemically split and then analyzed by NMR. Moreover, in a proof of principle we also studied PAO1 LPS when bound to gentamicin; this latter approach revealed interesting molecular information confirming the involvement of the O-antigen in the intake of the antibiotic by bacteria.

On this ground, we estimate that ssNMR approach is a very useful and very informative technique to observe the behavior of different LPSs in their intact chemical form and when bound to prokaryotic or eukaryotic ligands.

\section{RESULTS AND DISCUSSION}

Characterization of lipid A and core oligosaccharide from rough-type E. coli K12 LPS.

E. coli $\mathrm{K} 12$ cells were grown in media containing ${ }^{13} \mathrm{C}$ as the sole carbon source and LPS molecules extracted and purified (see Methods). Freeze dried LPS molecules were re-suspended in water and, prior to conducting the NMR analysis, the macromolecular state of LPS was investigated by electron microscopy. Spontaneously E. coli R-LPS assembled into vesicles of 100-200 nm diameter with lipid bilayers of $26+/-4 \mathrm{~nm}$ that were protected from staining (Figure 2).

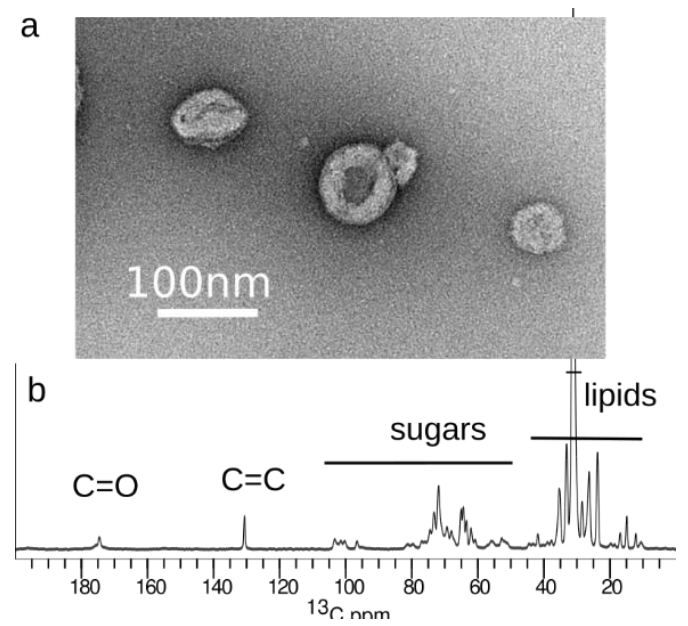

Figure 2 Characterization of E. coli R-LPS reconstituted in solution by a) Negative stain Electron 
microscopy and b) $1 \mathrm{D}{ }^{13} \mathrm{C}$ CP-MAS ssNMR spectrum recorded on a $600 \mathrm{MHz}$ spectrometer and at a spinning rate of $12.5 \mathrm{kHz}$.

The hydrated LPS has been then pelleted into a NMR rotor, of either 1.6 or $3.2 \mathrm{~mm}$ diameter and ssNMR experiments were recorded. Figure 2 shows a $1 \mathrm{D}{ }^{13} \mathrm{C}$ spectrum of $E$. coli R-LPS that features sharp NMR signals representing the different chemical groups of the LPS. To obtain site-specific information, we collected $2 \mathrm{D}{ }^{13} \mathrm{C}-{ }^{13} \mathrm{C}$ correlation experiments, exploiting either the dipolar coupling between the ${ }^{13} \mathrm{C}$ spins (using a Dipolar Assisted Rotational Resonance or DARR experiment) or the scalar coupling (with a heteronuclear Insensitive Nuclei Enhanced by Polarization Transfer INEPTbased experiment), respectively (Figures 3 and Supplementary Figure 1). While the former experiment was able to dissect the rigid moiety of the molecule, the latter was more sensitive to flexible parts of LPS (Supplementary Figure 1). Proton LPS resonances are too broad to be observed at low spinning speed but could be detected at $34 \mathrm{kHz}$ MAS rotation in a ${ }^{13} \mathrm{C}-{ }^{1} \mathrm{H}$ INEPT experiment.

a

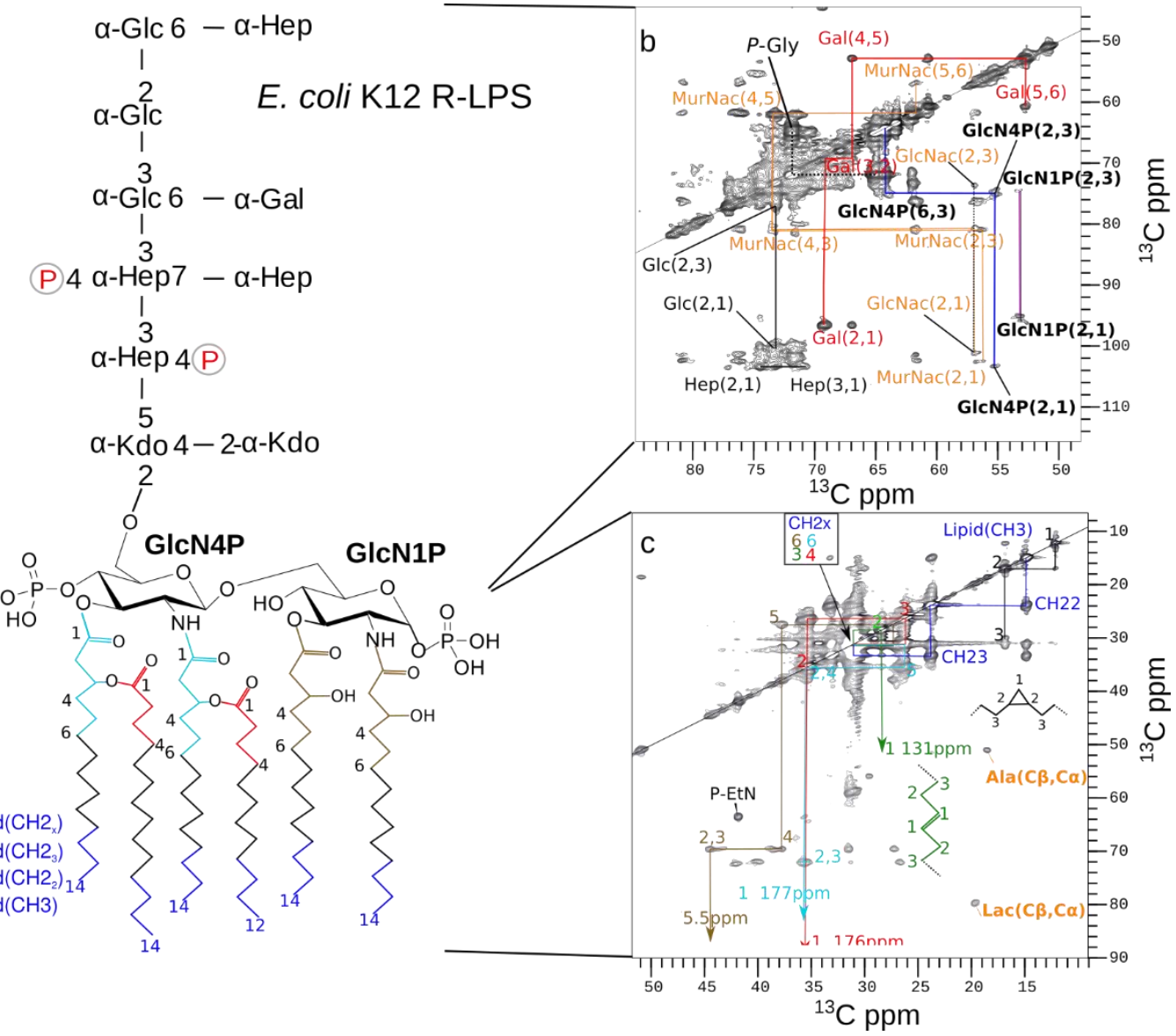

Figure 3 E. coli LPS Lipid A and core oligosaccharide characterization. a) Chemical structure of $E$. coli K12 R-LPS with ${ }^{13} \mathrm{C}-{ }^{13} \mathrm{C}$ DARR spectra focused on the sugar region or lipid region respectively (b and c). Assignment of peptidoglycan is shown in orange. Cyclopropane and insaturations are 
shown in c) in black and green respectively. $P$-EtN and $P$-Gly Phospholipids resonances are also indicated. $\mathrm{CH} 2 \mathrm{x}$ stands for $\mathrm{CH} 2$ at the middle of the lipid chains that are all chemically equivalent.

Resonances from most residues of E. coli LPS could be assigned and were in good agreement with those reported by liquid-state NMR (Supplementary Table 1). Signals for the two GlcN (glucosamine) of lipid A, straight assignments of the acyl parts of the molecules and parts of the core region including Kdo (keto-deoxyoctulosonate) and Hep (heptose) could be achieved (Figure 1). Lipid contaminants, probably tightly associated with LPS, were also identified, as cyclopropane modifications, normally present only on phospholipids. Insaturations, potentially occurring either on LPS fatty acids or on phospholipids acyl chains were assigned, as well as the two major phospholipids composing E. coli outer membrane inner leaflet, namely phosphatidyl ethanolamine and phosphatidyl glycerol. Peptidoglycan, another major component of the cell envelope, could also be detected, including GlcNAc and MurNAc (N-acetyl muramic acid) backbone sugars of the macromolecule, as well as characteristic Lactoyl signals (highlighted in yellow in figure 3). It is not unusual that peptidoglycan is associated with LPS preparation, presumably because of co-purification with Braun's lipoprotein that anchors PG in the outer membrane ${ }^{19}$.

Characterization of core oligosaccharide and O-antigen from P. aeruginosa PAO1.

Since rough type K-12 R-LPS from common laboratory strains could be successfully analyzed by ssNMR, we investigated a wild-type S-LPS extracted from the pathogenic P. aeruginosa PAO1 strain. This microbe is responsible for severe infections in immunocompromised patients and in particular in cystic fibrosis, and possesses variable LPS O-antigens ${ }^{20}$. Indeed, Pseudomonas LPS are composed of the same lipid A-core oligosaccharide skeleton but to which two different O-antigens are appended termed A and B bands ${ }^{20}$ (Supplementary Figure 1 and Figure 4 panel a). A-band Oantigen is based on a D-rhamnose repeating unit while B-band is based on a complex trisaccharide unit with two $\mathrm{N}$-acylated Mannuronic acids (ManNAc3NAcA or $3 \mathrm{AmA}$ ) and a FucNAc (Figure 4 panel a and Figure 5). These two O-antigen polysaccharides are simultaneously present at the surface 
of the bacteria.
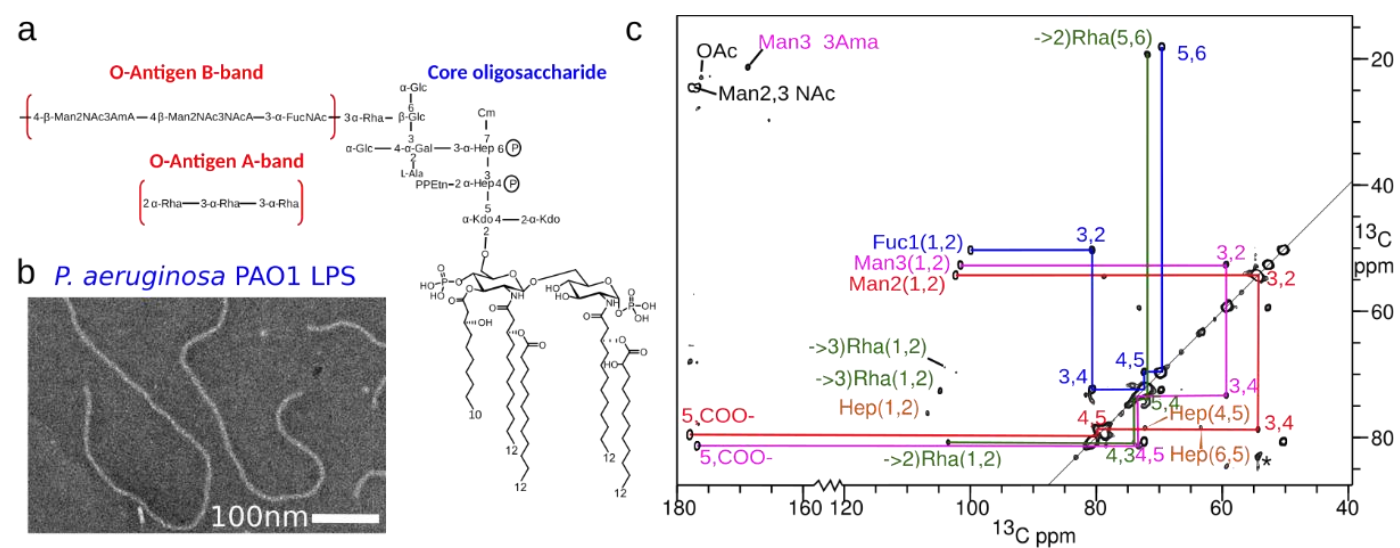

Figure 4 MAS ssNMR O-antigen from $P$. aeruginosa PAO1. a) Chemical structure of $P$. aeruginosa PAO1 S-LPS. b) EM negative stain micrograph of PAO1 LPS. c) extract of a ${ }^{13} \mathrm{C}-{ }^{13} \mathrm{C}$ Single Quantum $\mathrm{J}$ correlation spectrum of $P$. aeruginosa LPS with the assigned resonances showing clearly the Oantigen repeat (labeled Fuc1-Man2-Man3) and several resonances from sugars of the extended core. * Artefacts

${ }^{13}$ C-labeled PAO1 LPS was resuspended (see Methods) and first analyzed by Electron microscopy. The S-LPS was morphologically different from the E. coli R-LPS and formed elongated structures of various length of approximately $8 \mathrm{~nm}$ width (Figure 4 panel b). Formation of these elongated structures has been already observed for $N$. meningitis purified LPS ${ }^{21}$. Their shape suggested they assemble into elongated micelles, rather than vesicles, presumably because of their long hydrophilic O-antigen. The presence of the O-antigen might also explain the gel-like behavior of $P$. aeruginosa LPS in solution. Consequently, PAO1 LPS had to be centrifuged at 50000g overnight to be pelleted inside the NMR rotor.

Conversely to the E. coli R-LPS NMR experiments, only the last 4 carbons of the lipid chains of the PAO1 LPS could be observed (1 $\mathrm{CH}_{3}$ and $3 \mathrm{CH}_{2}$ see Supplementary Table 2$)$ and only few resonances were unambiguously assigned to the inner core oligosaccharides, namely to Kdo and heptose. Residues from the core were particularly visible in dipolar coupling-based experiments, suggesting they belonged to rigid regions of the LPS. Similarly, one rhamnose spin system with chemical shifts matching those of the outer core 3-substituted $\alpha$-Rha could be identified as well as alanine residues, probably bound to galactose residues (Figure 4) ${ }^{22}$.

The ssNMR analysis of $P$. aeruginosa LPS showed also the signals of the major B-band O- 
antigen trisaccharide $\alpha$-FucNAc- $\beta$-ManNAc3NAmA- $\beta$-ManNAc3NAcA repeating unit for which most resonances were unambiguously assigned (Figure 4 and Supplementary Figure 2). The Oantigen was also observed in dipolar-coupling based experiments thus suggesting a significant degree of order in the O-antigen chains, possibly through lateral contacts between O-antigen chains. These last data are in agreement with what observed with gel forming glycan polymers such as maltodextrins that exhibit the same behavior with a degree of order that allows to efficiently transfer the NMR magnetization through dipolar couplings using Cross Polarization (CP) experiments ${ }^{23}$.

D-rhamnose A-band repeating unit could not be detected unambiguously at a first glance since rhamnose also composes the outer core oligosaccharide common to both O-antigens. However, one sugar spin system with a lowfield displaced C-2 chemical shift could be unambiguously assigned to a 2-substituted rhamnose residue and cross peaks were assignable up to the methyl group (Figure 4 and Table S2). Several resonances, probably corresponding to 3-substituted rhamnose could be identified in the spectra but only up to the $\mathrm{C}-2 / \mathrm{H}-2$ groups, because of severe signals overlapping. Presence of A-Band polysaccharide could be confirmed by the presence of O-methylation $\left({ }^{13} \mathrm{C} /{ }^{1} \mathrm{H}\right.$ resonances at 57.4ppm/3.75ppm) characteristic of O-methyl rhamnose residue found in A-Band Oantigen $^{24}$. Nevertheless, this latter residue could not be assigned completely due to the lack of longrange distance correlations.

\section{Interaction of $P$. aeruginosa LPS with gentamicin antibiotic.}

LPS from PAO1 is a critical factor of virulence, which is particularly harmful in immunocompromised patients as those affected by cystic fibrosis. While A and B band O-antigens are found in patients at early onsets of the infection, only A band polysaccharide can be detected in chronic isolates of $P$. aeruginos $a^{25}$. Furthermore engineered $P$. aeruginosa $\mathrm{A}^{+} \mathrm{B}^{-}$bacteria were found to be less sensitive to gentamicin antibiotic, because of a higher affinity of gentamicin for bacteria presenting B-band O-antigen. Nevertheless, the molecular basis of mechanism remains unknown ${ }^{26,27}$. Based on such previous studies, we decided to investigate the effect of gentamicin on isolated and 
intact PAO1 LPS. Gentamicin is a natural antibiotic from the aminoglycosides family produced by M. echinospora, a Gram-positive bacterium (Figure 5). In vitro gentamicin very efficiently binds to isolated PAO1 LPS and this can be easily observed by solution NMR. Addition of LPS to gentamicin in solution leads to total disappearance of its NMR signals (Supplementary Figure 4), showing that all free gentamicin in solution have bound to LPS. We then used electron microscopy to assess the influence of gentamicin binding on LPS supramolecular organization. Addition of 25mM final concentration of gentamicin to PAO1 LPS did not significantly change the elongated micelles structures of isolated PAO1 LPS in solution (Figure 5 panel a).

a

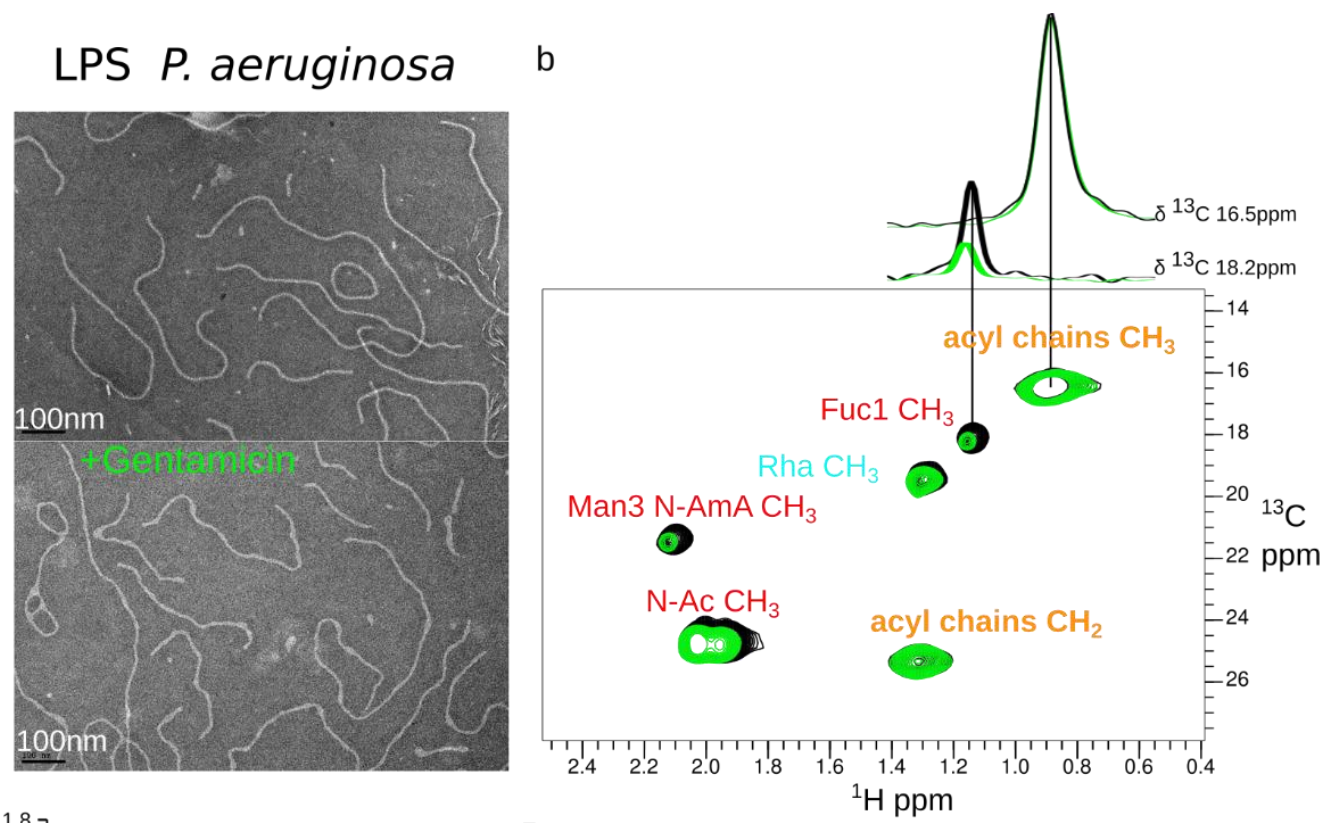

c 1,0

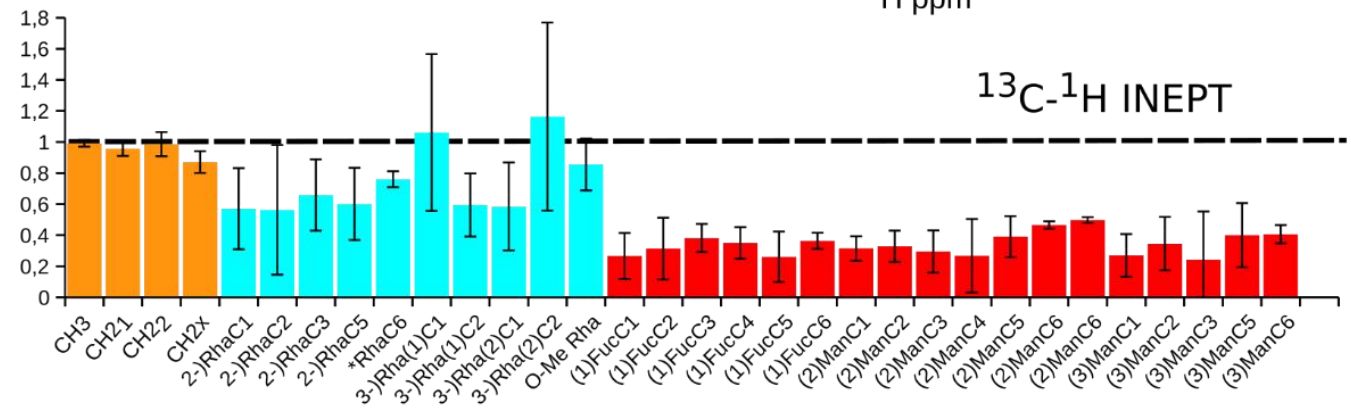

Lipid tail Rhamnose A-Band

O-antigen repeat

d

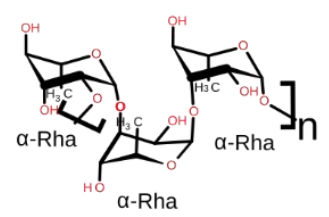

A-band LPS
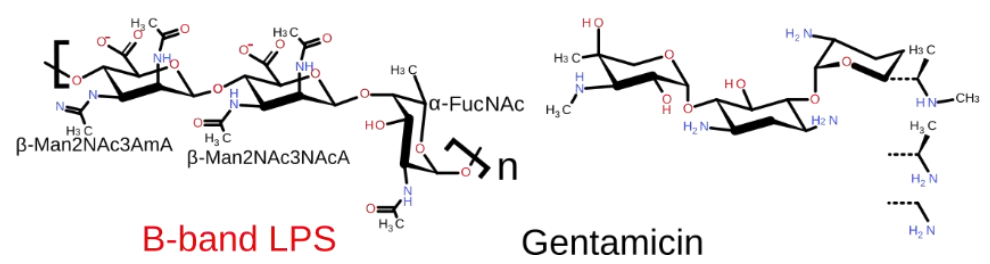

Gentamicin 
Figure $5 P$. aeruginosa LPS O-antigen is affected by interaction with gentamicin. a) EM pictures with negative staining of $P$. aeruginosa LPS in absence (up) or presence of $25 \mathrm{mM}$ Gentamicin (down). b) ${ }^{13} \mathrm{C}-{ }^{1} \mathrm{H}$ INEPT ssNMR spectrum of LPS in absence or presence (green) of $25 \mathrm{mM}$ gentamicin. Spectra are normalized to the intensity of lipid $\mathrm{CH}_{3}$ peak. 1D slices of Lipid $\mathrm{A} \mathrm{CH}_{3}$ and fucose $\mathrm{CH}_{3}$ peaks are shown above the spectrum. c) Ratio of peak intensities $\mathrm{I}_{\text {gentamicin }} \mathrm{I}_{0}$ measured in the ${ }^{13} \mathrm{C}-{ }^{1} \mathrm{H}$ INEPT experiment. d) Chemical structures of B-Band, A-Band trisaccharide repeats and of gentamicin.

In absence of supramolecular reorganization, ssNMR approach was used to investigate the gentamicin interaction with LPS at an atomic resolution. $25 \mathrm{mM}$ gentamicin was added to a $40 \mathrm{mg} / \mathrm{ml}$ PAO1 LPS solution which was ultracentrifuged into the NMR rotor (see Methods). ${ }^{13} \mathrm{C}-{ }^{13} \mathrm{C} \mathrm{J}-$ correlation experiments as well as ${ }^{13} \mathrm{C}-{ }^{1} \mathrm{H}$ INEPT experiments were thus recorded at $34 \mathrm{kHz} \mathrm{MAS}$ in presence of gentamicin. Gentamicin bound LPS produced a similar spectrum as free PAO1 LPS, showing mostly the O-antigen repeats and the last $4 \mathrm{CH}$ groups of the lipid A part. Small chemical shift variations are observed for the polysaccharides resonances (Supplementary Figure 5), but not for the resonances of the lipid A (Figure 5 panel b). Comparison of the peak intensities before and after addition of gentamicin also reveals that the lipid A resonances are not affected by the gentamicin interaction (Figure 5 panel b). However, polysaccharide resonances of the O-antigen are significantly decreased in intensity in presence of gentamicin. Interestingly, the presence of gentamicin has even a stronger effect on the resonance intensities corresponding to the B-band polysaccharides relative to the saccharides of the A-Band (Figure 5 panels b and c).

This specific decrease in intensity observed for the O-antigen, and more specifically for the saccharide present in the O-antigen B-Band can be interpreted as an interaction of gentamicin with some specific region of the LPS. To analyze in more detail the effect of gentamicin on the local flexibility of the LPS, we have also measured different relaxation parameters. However, only ${ }^{13} \mathrm{C} \mathrm{T}_{1}$ longitudinal relaxation constant time can be measured with a sufficient sensitivity and in a reasonable experimental time (Supplementary Figure 4). Unfortunately, these longitudinal relaxation rates do not show significant variations and cannot be used to refine the effect of the gentamicin on the Oantigen flexibility.

Though we have information on individual B-band trisaccharide resonances we could not 
isolate a unique residue or chemical group that was specifically affected by gentamicin interaction and we can conclude that the whole repeating unit is a determinant of the interaction. In agreement, we can speculate that the B-band possesses two negative charges (the two Mannuronic acids) per repeating unit which should bridge with the free amino groups of gentamicin, i.e., ammonium groups at physiological pH (Figure 5 panel d). Furthermore, additional contacts could be assumed by the several ester and amide carbonyl groups of the O-antigen with the same positively charged groups of gentamicin. Thus, the specific binding effect that we observed between gentamycin and the B-Band O-antigen explains why the PAO1 LPS B band positive strains are sensitive to gentamicin whereas A-band strains are not. Due to avidity phenomena, it can be certainly deemed that this binding process increases local concentration of gentamicin at outer membrane and increases gentamicin lethality on B band possessing strains.

\section{CONCLUSION}

The direct observation of LPS endotoxins in their natural environment constitutes an important step in the field of molecular microbiology and, in particular, toward the understanding of interactions of microbes with their eukaryotic hosts, either beneficial or harmful. Many studies have been published so far in which the double role and the action of the endotoxins has been elucidated, i.e., either as barriers to antibiotics or as elicitors/suppressors of innate immunity in eukaryotes ${ }^{1,2,28,29}$. Beside this, however, very rarely it has been possible to understand the supramolecular behavior and their action as whole molecule, their delivery to the receptors or their action against antibiotics. Nowadays, their study is still confined to a complex operation of bench organic and analytical bioorganic chemistry ${ }^{7}$ with no chances to comprehend how the primary structure affects the overall 3D structure. It is in fact pivotal to study LPS interactions with eukaryotic and prokaryotic lectins, immunity proteins (TLR4/MD2, caspases) and symbiosis proteins and get a perspective at molecular scale of the whole intact molecule when bound and/or recognized by other ligands.

In this work we have employed ssNMR for studying two different and archetypal LPS, with 
either long or short carbohydrate chain, in a membrane like structure without any alteration of the molecules, in the absence of detergents and in suitable conditions to study LPS interactions. Furthermore, this study further confirmed the capacity of this approach to gain access to parts of the macromolecule that present different flexibilities (Figure 6). MAS ssNMR allowed to either record experiments on the most flexible segments of a molecule through experiments similar as those recorded in liquid-state, or on the most rigid parts by Cross-Polarization experiments. Comparison of the two types of experiments in Figure 6 point out an efficient CP transfer on R-LPS that shows mostly the relatively rigid lipid and core oligosaccharides while CP transfer is less efficient in S-LPS where the spectrum is dominated by flexible O-antigen. The versatility of the method will be particularly efficient in studying LPSs ligand binding, and especially binding to large ligands such as proteins which will significantly rigidify the LPS or when the rigid lipid A part is directly involved in the binding with a receptor (TLR4/MD2, CD14 or LBP protein binding). Further advances in ssNMR methods, in particular higher magic-angle spinning frequencies permit ${ }^{1} \mathrm{H}$ detection experiments with high resolution and increased sensitivity. High speed spinning (100-111kHz) allows to record 2D and 3D experiments through dipolar or J-couplings similar to liquid state experiments , and on complex ${ }^{13} \mathrm{C} /{ }^{15} \mathrm{~N} /\left({ }^{2} \mathrm{H}\right)$ labeled protein systems and on unlabeled molecules with together very low amounts of sample ${ }^{30}$ and short experimental times. This should prove particularly useful for the study of LPS alone or in interaction with ligands and thus contribute to the elucidation of the fundamental properties of outer membrane permeability.

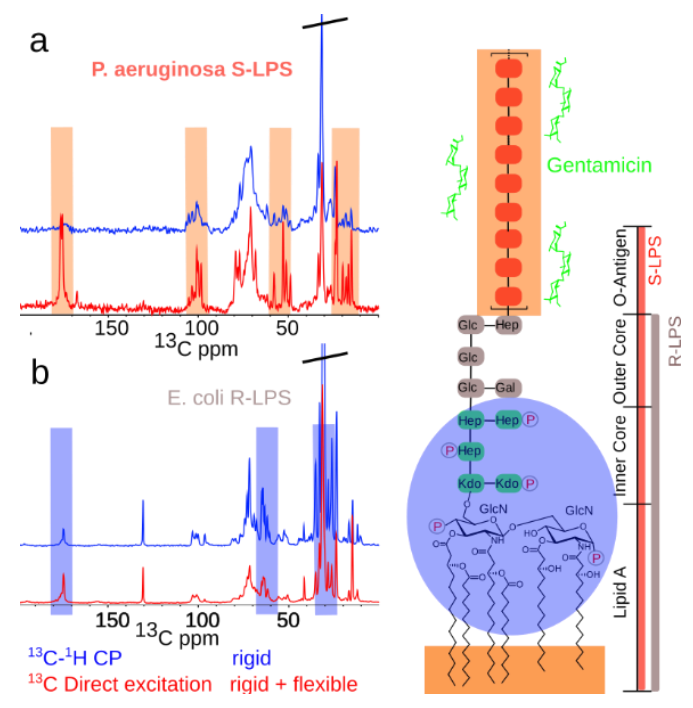


Figure 6 ssNMR highlights regions of different flexibilities and regions of interactions in LPS.

a) Comparison of $1 \mathrm{D}{ }^{13} \mathrm{C}$ Spectra of LPS with direct ${ }^{13} \mathrm{C}$ excitation (red) or through ${ }^{13} \mathrm{C}-{ }^{1} \mathrm{H}$ Cross polarization experiment(blue) shows that $P$. aeruginosa S-LPS, that assemble into micelles, displays mainly flexible signals (orange) belonging to O-antigens with less efficient CP transfer. These regions were identified as interacting with gentamicin antibiotic.

b) E. coli R. LPS in vesicles CP spectrum shows rigid portions of the LPS (blue), mainly core oligosaccharides and lipids.

\section{METHODS}

\section{Cell growths and LPS extraction}

LPS ${ }^{13}$ C-labeling was performed by growing E. coli BL21(DE3) or P. aeruginosa PA01 in M9 minimal medium ${ }^{31}$ supplemented with $0.1 \%(\mathrm{w} / \mathrm{v}){ }^{13} \mathrm{C}$ glucose, as the sole carbon source. Cells were harvested from 1 liter of bacterial culture grown to $\mathrm{OD}_{600}$ of 0.8 and the bacterial pellet was lyophilized, washed with distilled water, ethanol and acetone followed by ultracentrifugation steps (45,000 rpm, $\left.4{ }^{\circ} \mathrm{C}, 16 \mathrm{~h}\right)$. LPS were extracted by hot phenol/water extraction ${ }^{32}$. Both phases were dialyzed and lyophilized, followed by protease and nuclease digestions, then extensive dialysis against water and freeze-drying. Presence of LPS was assessed by SDS-PAGE all along the purification. Gentamicin was obtained from SIGMA(G1264).

\section{Electron microscopy}

Samples of LPS at 0.1 to $1 \mathrm{mg} / \mathrm{ml}$ concentration in water for E. coli LPS or 50mM MES pH 6.0 for P. aeruginosa PAO1 LPS were used. LPS PAO1 with gentamicin was studied at $0.1 \mathrm{mg} / \mathrm{ml}$ in presence of $25 \mathrm{mM}$ gentamicin. Samples were absorbed to the clean side of a carbon film on mica, stained with Sodium Silico Tungstate and transferred to a 400-mesh copper grid. The images were taken under low dose conditions $\left(<10 \mathrm{e}^{-} / \AA^{2}\right)$ at a magnification of $23 \mathrm{Kx}$ and $49 \mathrm{Kx}$ times with defocus values between 1.2 and $2.5 \mu \mathrm{m}$ on a Tecnai $12 \mathrm{LaB} 6$ electron microscope at $120 \mathrm{kV}$ accelerating voltage using CCD Camera Gatan Orius 1000. 


\section{Nuclear Magnetic Resonance}

Lyophylised LPS sample was resuspended at $\sim 50 \mathrm{mg} / \mathrm{ml}$ final concentration in Milli Q water for $E$. coli at LOS or 50mM MES pH 6.0 for P. aeruginosa PAO1 LPS. E. coli LOS was sedimented into rotors by centrifugation $\left(16-50.10^{3} \mathrm{~g}\right)$ for 5 minutes. P. aeruginosa LPS was sedimented into rotors at 50000g for 16 hours. NMR experiments were recorded on Agilent 600MHz VNMRS spectrometer equipped with MAS $3.2 \mathrm{~mm}$ or $1.6 \mathrm{~mm}$ HCN probes. MAS frequencies used were $12.5 \mathrm{kHz}$ when employing $3.2 \mathrm{~mm}$ probe and $34 \mathrm{kHz}$ with the $1.6 \mathrm{~mm}$ probe. The sample temperature was set to ca. $25^{\circ} \mathrm{C}$. For $2 \mathrm{D}{ }^{13} \mathrm{C}-{ }^{13} \mathrm{C}$ correlation experiments acquisition times were typically $14-20 \mathrm{~ms}$ for direct dimension and 5-7 ms in the indirect dimensions, interscan delay was set to 2-4 seconds. ${ }^{1} \mathrm{H}$ SPINAL64 decoupling with a radio-frequency (rf) of 100-110 kHz was applied. In CP experiments, the ${ }^{1} \mathrm{H}$ radiofrequency field was between 55 and $60 \mathrm{kHz}$, and the ${ }^{13} \mathrm{C}$ rf field adjusted to match the HartmannHahn condition with $n=1$, and the $\mathrm{CP}$ time was set between 0.5 and $1.5 \mathrm{~ms} .1 \mathrm{D}{ }^{13} \mathrm{C}$ experiments with direct ${ }^{13} \mathrm{C}$ excitation or ${ }^{1} \mathrm{H}-{ }^{13} \mathrm{C}$ CP-MAS were recorded with $12-19$ ms acquisition time and an interscan delay of 2-3 seconds. Typically, ${ }^{13} \mathrm{C}-{ }^{13} \mathrm{C}$ DARR experiment on E. coli LPS in $3.2 \mathrm{~mm}$ rotor was acquired in 41 hours with a $100 \mathrm{~ms}$ mixing time and ${ }^{13} \mathrm{C}-{ }^{13} \mathrm{C}$ Single Quantum J experiment in 16 hours. ${ }^{13} \mathrm{C}-{ }^{1} \mathrm{H}$ INEPT experiments ( $\mathrm{T}_{\text {inept }}=1 \mathrm{~ms}$ ) and 2D ${ }^{13} \mathrm{C}-{ }^{13} \mathrm{C}$ Single Quantum J experiment of PAO1 LPS in 1.6mm rotor were recorded in 6 and 24 hours respectively. A PAO1 LPS sample at 40mg/ml in 50mM MES pH 6.0 was split in two and gentamicin at 25mM final concentration was added to one of the sample and they were ultracentrifuged into NMR rotor as described above. ${ }^{13} \mathrm{C}$ $\mathrm{T} 1$ longitudinal relaxation time constants were obtained with series of ${ }^{13} \mathrm{C}-{ }^{1} \mathrm{H}$ INEPT experiments at $34 \mathrm{kHz}$ with inversion recovery periods and delays from $1 \mathrm{~ms}$ to $1.3 \mathrm{~s}$ in absence and presence of gentamicin. $\mathrm{T}_{1}$ values were fitted with CcpNmr software and presented with the fitting error provided by the software. NMR experiments were processed with NMRPipe ${ }^{33}$ or Topspin 3.5 and analysed with CcpNmr 2.42 software ${ }^{34}$. Comparison of $1 \mathrm{D}{ }^{13} \mathrm{C}$ Spectra of LPS with direct ${ }^{13} \mathrm{C}$ excitation or through ${ }^{13} \mathrm{C}-{ }^{1} \mathrm{H}$ Cross polarization experiment Spectra were recorded at $600 \mathrm{MHz}$ and at a spinning 
rate of $12.5 \mathrm{kHz}$ with identical numbers of scans for each sample (PAO1-LPS 128, K12 LPS 64), and baseline was shifted for improved clarity.

\section{ACKNOWLEDGEMENTS}

This work was supported by the Train2Target project granted from the European Union's Horizon 2020 framework program for research and innovation (Project \#721484) and partly supported by the French National Research Agency in the framework of the " Investissements d'avenir” program Glyco@Alps (ANR-15-IDEX-02). This work used the platforms of the Grenoble Instruct centre (ISBG; UMS 3518 CNRS-CEA-UJF-EMBL) with support from FRISBI (ANR-10-INSB-05-02) and GRAL (ANR-10-LABX-49-01) within the Grenoble Partnership for Structural Biology (PSB). The electron microscope facility is supported by the Rhône-Alpes Region, the Fondation Recherche Medicale (FRM), the fonds FEDER, the Centre National de la Recherche Scientifique (CNRS), the CEA, the University of Grenoble, EMBL, and the GIS-Infrastrutures en Biologie Sante et Agronomie (IBISA). We thank D. Fenel and G. Schoehn, from the Electron Microscopy platform of the Integrated Structural Biology of Grenoble (ISBG, UMI3265). We gratefully thank M. Richerd for great assistance in spectra analysis and C. Bougault and B. Bersch for stimulating discussions.

\section{ASSOCIATED CONTENT}

Supporting information is available including NMR chemical shifts table and additional NMR spectra: This material is available free of charge via the Internet at http://pubs.acs.org.

The authors declare no competing financial interest.

Bibliography

1. Brodsky, I. E., and Medzhitov, R. (2009) Targeting of immune signalling networks by bacterial 
pathogens. Nat. Cell Biol. 11, 521-526.

2. Silipo, A., Erbs, G., Shinya, T., Maxwell Dow, J. M., Parrilli, M., Lanzetta, R., Shibuya, N., Newman, M. A., and Molinaro, A. (2009) Glycoconjugates as elicitors or suppressors of plant innate immunity. Glycobiology 20, 406-419.

3. Whitfield, C., and Trent, M. S. (2014) Biosynthesis and Export of Bacterial Lipopolysaccharides. Annu. Rev. Biochem. 83, 99-128.

4. Delcour, A. H. (2009) Outer membrane permeability and antibiotic resistance. Biochim. Biophys. Acta 1794, 808-16.

5. Molinaro, A., Holst, O., Lorenzo, F. Di, Callaghan, M., Nurisso, A., D’Errico, G., Zamyatina, A., Peri, F., Berisio, R., Jerala, R., Jiménez-Barbero, J., Silipo, A., and Martín-Santamarí, S. (2015) Chemistry of lipid a: At the heart of innate immunity. Chem. - A Eur. J. 21, 500-519.

6. Yang, J., Zhao, Y., and Shao, F. (2015) Non-canonical activation of inflammatory caspases by cytosolic LPS in innate immunity. Curr. Opin. Immunol. 32, 78-83.

7. De Castro, C., Parrilli, M., Holst, O., and Molinaro, A. (2010) Microbe-Associated Molecular Patterns in Innate Immunity: Extraction and Chemical Analysis of Gram-Negative Bacterial Lipopolysaccharides. Methods Enzymol. 480, 89-115.

8. DiLorenzo, F., Palmigiano, A., Duda, K. A., Pallach, M., Busset, N., Sturiale, L., Giraud, E., Garozzo, D., Molinaro, A., and Silipo, A. (2017) Structure of the Lipopolysaccharide from the Bradyrhizobium sp. ORS285 rfaL Mutant Strain. ChemistryOpen 6, 541-553.

9. Renault, M., Tommassen-van Boxtel, R., Bos, M. P., Post, J. A., Tommassen, J., and Baldus, M. (2012) Cellular solid-state nuclear magnetic resonance spectroscopy. Proc. Natl. Acad. Sci. 109, $4863-4868$.

10. Ilg, K., Zandomeneghi, G., Rugarabamu, G., Meier, B. H., and Aebi, M. (2013) HR-MAS NMR reveals a pH-dependent LPS alteration by de-O-acetylation at abequose in the O-antigen of Salmonella enterica serovar Typhimurium. Carbohydr. Res. 382, 58-64.

11. Johnson, C. L., Ridley, H., Marchetti, R., Silipo, A., Griffin, D. C., Crawford, L., Bonev, B., 
Molinaro, A., and Lakey, J. H. (2014) The antibacterial toxin colicin N binds to the inner core of lipopolysaccharide and close to its translocator protein. Mol. Microbiol. 92, 440-452.

12. Wang, W., Sass, H. J., Zähringer, U., and Grzesiek, S. (2008) Structure and dynamics of 13C,15Nlabeled lipopolysaccharides in a membrane mimetic. Angew. Chem. Int. Ed. Engl. 47, 9870-9874.

13. Zähringer, U., Ittig, S., Lindner, B., Moll, H., Schombel, U., Gisch, N., and Cornelis, G. R. (2014) NMR-based structural analysis of the complete rough-type lipopolysaccharide isolated from Capnocytophaga canimorsus. J. Biol. Chem. 289, 23963-23976.

14. Kern, T., Hediger, S., Müller, P., Giustini, C., Joris, B., Bougault, C., Vollmer, W., and Simorre, J. P. (2008) Toward the characterization of peptidoglycan structure and protein-peptidoglycan interactions by solid-state NMR spectroscopy. J. Am. Chem. Soc. 130, 5618-5619.

15. Kern, T., Giffard, M., Hediger, S., Amoroso, A., Giustini, C., Bui, N. K., Joris, B., Bougault, C., Vollmer, W., and Simorre, J. P. (2010) Dynamics characterization of fully hydrated bacterial cell walls by solid-state NMR: Evidence for cooperative binding of metal ions. J. Am. Chem. Soc. 132, 10911-10919.

16. Schanda, P., Triboulet, S., Laguri, C., Bougault, C. M., Ayala, I., Callon, M., Arthur, M., and Simorre, J. P. (2014) Atomic model of a cell-wall cross-linking enzyme in complex with an intact bacterial peptidoglycan. J. Am. Chem. Soc. 136, 17852-17860.

17. Cegelski, L., Kim, S. J., Hing, A. W., Studelska, D. R., O’Connor, R. D., Mehta, A. K., and Schaefer, J. (2002) Rotational-echo double resonance characterization of the effects of vancomycin on cell wall synthesis in Staphylococcus aureus. Biochemistry 41, 13053-13058.

18. Warnet, X. L., Arnold, A. A., Marcotte, I., and Warschawski, D. E. (2015) In-Cell Solid-State NMR: An Emerging Technique for the Study of Biological Membranes. Biophys. J. 109, 2461-2466. 19. Vollmer, W., Blanot, D., and De Pedro, M. A. (2008) Peptidoglycan structure and architecture. FEMS Microbiol. Rev. 32, 149-167.

20. Knirel, Y. A., Bystrova, O. V., Kocharova, N. A., Zähringer, U., and Pier, G. B. (2006) Conserved and variable structural features in the lipopolysaccharide of \&lt;I\&gt;Pseudomonas 
aeruginosa\&lt;/I\&gt; J. Endotoxin Res. 12, 324-336.

21. Bjerre, A., Brusletto, B., Mollnes, T. E., Fritzsønn, E., Rosenqvist, E., Wedege, E., Namork, E., Kierulf, P., and Brandtzaeg, P. (2002) Complement activation induced by purified Neisseria meningitidis lipopolysaccharide (LPS), outer membrane vesicles, whole bacteria, and an LPS-free mutant. J. Infect. Dis. 185, 220-228.

22. Di Lorenzo, F., Silipo, A., Bianconi, I., Lore’, N. I., Scamporrino, A., Sturiale, L., Garozzo, D., Lanzetta, R., Parrilli, M., Bragonzi, A., and Molinaro, A. (2015) Persistent cystic fibrosis isolate Pseudomonas aeruginosa strain RP73 exhibits an under-acylated LPS structure responsible of its low inflammatory activity. Mol. Immunol. 63, 166-175.

23. van Duynhoven, J. P. ., Kulik, A. ., Jonker, H. R. ., and Haverkamp, J. (1999) Solid-like components in carbohydrate gels probed by NMR spectroscopy. Carbohydr. Polym. 40, 211-219.

24. Arsenault, T., Hughes, D. W., and MacLean, D. B. (1991) Structural studies on the polysaccharide portion of “A-band” lipopolysaccharide from a mutant (AK1401) of Pseudomonas aeruginosa strain PAO1. Can. J. Chem. 69, 1273-1280.

25. Rocchetta, H. L., Burrows, L. L., and Lam, J. S. (1999) Genetics of O-antigen biosynthesis in Pseudomonas aeruginosa. Microbiol. Mol. Biol. Rev. 63, 523-53.

26. Kadurugamuwa, J. L., Lam, J. S., and Beveridge, T. J. (1993) Interaction of gentamicin with the A band and B band lipopolysaccharides of Pseudomonas aeruginosa and its possible lethal effect. Antimicrob. Agents Chemother. 37, 715-721.

27. Makin, S. A., and Beveridge, T. J. (1996) Pseudomonas aeruginosa PAO1 ceases to express serotype-specific lipopolysaccharide at 45??C. J. Bacteriol. 178, 3350-3352.

28. Stokes, J. M., MacNair, C. R., Ilyas, B., French, S., Côté, J.-P., Bouwman, C., Farha, M. A., Sieron, A. O., Whitfield, C., Coombes, B. K., and Brown, E. D. (2017) Pentamidine sensitizes Gramnegative pathogens to antibiotics and overcomes acquired colistin resistance. Nat. Microbiol. 2, 17028.

29. May, J. M., Owens, T. W., Mandler, M. D., Simpson, B. W., Lazarus, M. B., Sherman, D. J., 
Davis, R. M., Okuda, S., Massefski, W., Ruiz, N., and Kahne, D. (2017) The Antibiotic Novobiocin Binds and Activates the ATPase That Powers Lipopolysaccharide Transport. J. Am. Chem. Soc. 139, $17221-17224$.

30. Quinn, C. M., and Polenova, T. (2017) Structural biology of supramolecular assemblies by magicAngle spinning NMR spectroscopy. Q. Rev. Biophys.

31. Laguri, C., Sperandeo, P., Pounot, K., Ayala, I., Silipo, A., Bougault, C. M., Molinaro, A., Polissi, A., and Simorre, J. P. (2017) Interaction of lipopolysaccharides at intermolecular sites of the periplasmic Lpt transport assembly. Sci. Rep. 7, 9715.

32. Galanos, C., Lüderitz, O., and Westphal, O. (1969) A New Method for the Extraction of R Lipopolysaccharides. Eur. J. Biochem. 9, 245-249.

33. Delaglio, F., Grzesiek, S., Vuister, G. W., Zhu, G., Pfeifer, J., and Bax, A. (1995) NMRPipe: a multidimensional spectral processing system based on UNIX pipes. J. Biomol. NMR 6, 277-93.

34. Vranken, W. F., Boucher, W., Stevens, T. J., Fogh, R. H., Pajon, A., Llinas, M., Ulrich, E. L., Markley, J. L., Ionides, J., and Laue, E. D. (2005) The CCPN data model for NMR spectroscopy: Development of a software pipeline. Proteins Struct. Funct. Genet. 59, 687-696. 